\title{
Thermoelectric power of ferroelectric potassium vanadate, cesium vanadate, lithium vanadate and their solid solutions
}

\author{
A J KULKARNI ${ }^{\dagger}$, S P RASAL ${ }^{\dagger \dagger}$, T S MAGDUM ${ }^{\dagger \dagger \dagger}$ and \\ S H CHAVAN* \\ Department of Physics, Shivaji University, Kolhapur 416004, India \\ ${ }^{+}$Walchand Institute of Technology, Solapur 413006, India \\ ${ }^{t \dagger}$ Mudhoji College, Phaltan 415 523, India \\ ${ }^{++\dagger}$ Vivekanand College, Kolhapur 416002, India
}

MS received 15 June 1992; revised 4 January 1993

\begin{abstract}
The thermoelectric power of the vanadates of potassium, cesium and lithium and their solid solutions was measured in the temperature range covering their transition points. The thermoelectric power measurement was carried out by the two-electrode technique for pellets of polycrystalline ceramic samples. The thermoelectric power increased with temperature initially, then decreased attaining a zero value at the transition temperature. As the concentration of $\mathrm{KVO}_{3}$ increased the thermoelectric power decreased for the solid solutions $\left(\mathrm{K}_{x}-\mathrm{Cs}_{1-x}\right) \mathrm{VO}_{3}$, whereas the thermoelectric power increased with increase in concentration of $\mathrm{KVO}_{3}$ for the solid solutions $\left(\mathrm{K}_{x}-\mathrm{Li}_{1-x}\right) \mathrm{VO}_{3}$. Vanadates of potassium, cesium, and lithium and their solid solutions showed $P$ type semiconductor behaviour in ferroelectric state and $n$ type semiconductor behaviour in paraelectric region.
\end{abstract}

Keywords. Thermoelectric power; phase transition; vanadate solid solutions.

\section{Introduction}

Studies on thermoelectric power and d.c. electrical conductivity give fundamental information on the mechanism of conduction in solids. Gran Ville and Hogarth (1951) and Bloem (1956) found that a rough measurement of thermo-emf can be surprisingly accurate and therefore it is sufficient to determine the sign of the charge carriers. Heikes (1961) observed that the Seebeck coefficient $(S)$ is independent of temperature and that its magnitude basically depends upon the density of the charge carriers. Austin and Mott (1969) and Bosman and Daal (1970) stated that along with electrical conductivity data, thermoelectric power can also give information on the drift mobility of the charge carriers and electrical conduction mechanism in solids. Yadav et al (1985) studied the thermoelectric power of $\mathrm{PrVO}_{4}$ single crystals. The electrical conductivity and thermoelectric power of light and heavy rare earth vanadates were studied by Gaur and Lal $(1985,1986)$. Electrical transport properties of polycrystalline cromium vanadate were investigated by Gupta et al (1987). The temperature dependence of thermoelectric power of alkali metals was studied by Srivastava et al (1990).

\footnotetext{
* To whom all correspondence should be addressed.
} 


\section{Experimental set-up and procedures}

\subsection{Sample preparation}

A stoichiometric mixture of alkali meta carbonates and vanadium pentoxide was heated slowly inside a globar furnace up to $750^{\circ} \mathrm{C}$ for $4 \mathrm{~h}$ and then melt furnace-cooled to room temperature to obtain crystalline vanadates. The samples of the solid solutions $\left(\mathrm{K}_{x}-\mathrm{Cs}_{1-x}\right) \mathrm{VO}_{3}$ were prepared from the respective vanadates with molar proportions $(x=0.2,0.4,0.6,0.8)$ and heated in a globar furnace at $750^{\circ} \mathrm{C}$ for $4 \mathrm{~h}$ in a platinum crucible and then cooled to room temperature. Similarly, the solid solutions of $\left(\mathrm{K}_{x}-\mathrm{Li}_{1-x}\right) \mathrm{VO}_{3}(x=0.2,0.4,0.6,0.8)$ were prepared by taking the appropriate molar percentage of potassium and lithium vanadates. The samples prepared were confirmed by using scanning $X$-ray diffractometer. The pellets of the samples (measuring $1 \mathrm{~cm}$ diam. and $0.2 \mathrm{~cm}$ thickness) were prepared under a pressure of $7.4 \times 10^{7} \mathrm{~kg} \mathrm{~m}^{-2}$. The pellets thus prepared were sintered on a platinum foil at $500^{\circ} \mathrm{C}$ for $3 \mathrm{~h}$. The opposite faces of the test samples were coated with thin layers of air-drying silver paste to have good electrical contacts with the stainless steel electrodes of the sample holder fabricated in our laboratory.

\subsection{Experimental}

The experimental apparatus consists of an electrically heated furnace, a temperature controller arrangement, a digital d.c. microvoltmeter, VMV 15 (Vasavi Electronics), a specially fabricated sample holder and a digital multimeter.

To measure thermoelectric power, the samples of $\mathrm{KVO}_{3}, \mathrm{CsVO}_{3}, \mathrm{LiVO}_{3}$ and their solid solutions viz. $\left(\mathrm{K}_{x}-\mathrm{Cs}_{1-x}\right) \mathrm{VO}_{3}$ and $\left(\mathrm{K}_{x}-\mathrm{Li}_{1-x}\right) \mathrm{VO}_{3}(x=0.2,0.4,0.6$ and 0.8 ) were used in the form of pellets.

The thermoelectric power of these samples was measured by producing a thermal gradient $\left(\Delta T=25^{\circ} \mathrm{C}\right)$ across the samples using a small heater attached to one of the hard electrodes of the sample holder. Silver electrodes were used for the present investigation. Sufficient time was given after applying thermal gradient to get stability before recording thermo-emf $(\Delta E)$. The samples were heated by establishing the constant thermal gradient $\left(\Delta T=25^{\circ} \mathrm{C}\right)$ and the developed thermo-emf $(\Delta E)$ was measured at different ambient temperatures of the samples. The thermoelectric power (S) was determined by using the relation, $S=\Delta E / \Delta T$.

\section{Results and discussion}

The variation of thermoelectric power with temperature of $\mathrm{KVO}_{3}, \mathrm{CsVO}_{3}$, and $\mathrm{LiVO}_{3}$ in their ferroelectric and paraelectric regions is represented in figure 1. Similarly, figures 2 and 3 indicate the relationship of thermoelectric-emf with temperature for the solid solutions $\left(\mathrm{K}_{x}-\mathrm{Cs}_{1-x}\right) \mathrm{VO}_{3}$ and $\left(\mathrm{K}_{x}-\mathrm{Li}_{1-x}\right) \mathrm{VO}_{3}$ respectively, where $(x=0.2,0.4,0.6$ and 0.8$)$. Figures $1-3$ show that $S$ increases with increase in temperature. The figures also reveal that $S$ decreased with increase in temperature in the high temperature region, and became negative in paraelectric region. The transition of $S$ from positive to negative value took place at the curie temperature. After the transition temperature, it again increased but in the reverse 


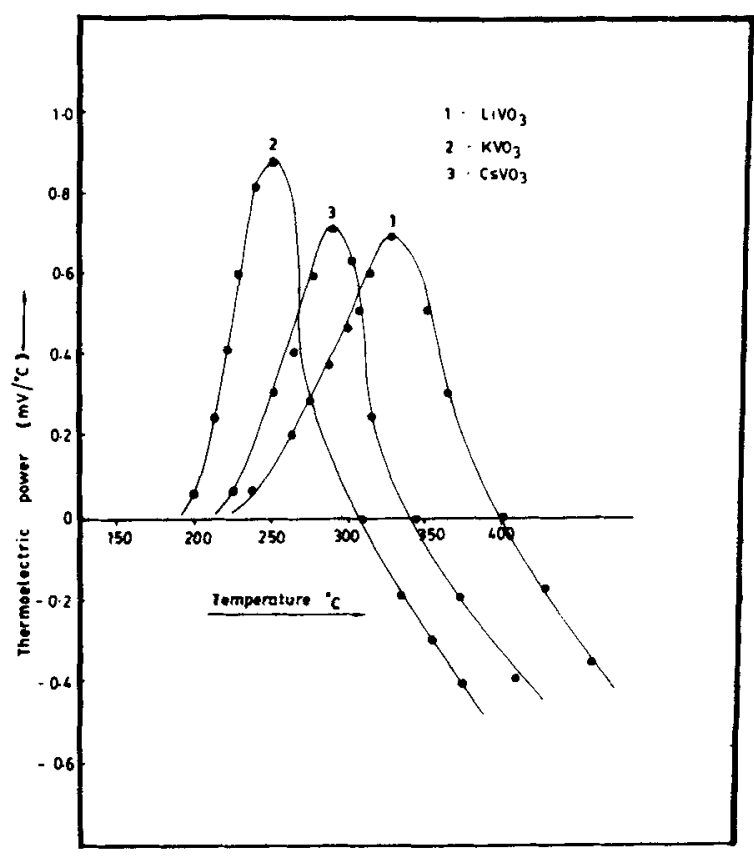

Figure 1. Variation of thermoelectric power $\left(\mathrm{mV} /{ }^{\circ} \mathrm{C}\right)$ with temperature $\left({ }^{\circ} \mathrm{C}\right)$.

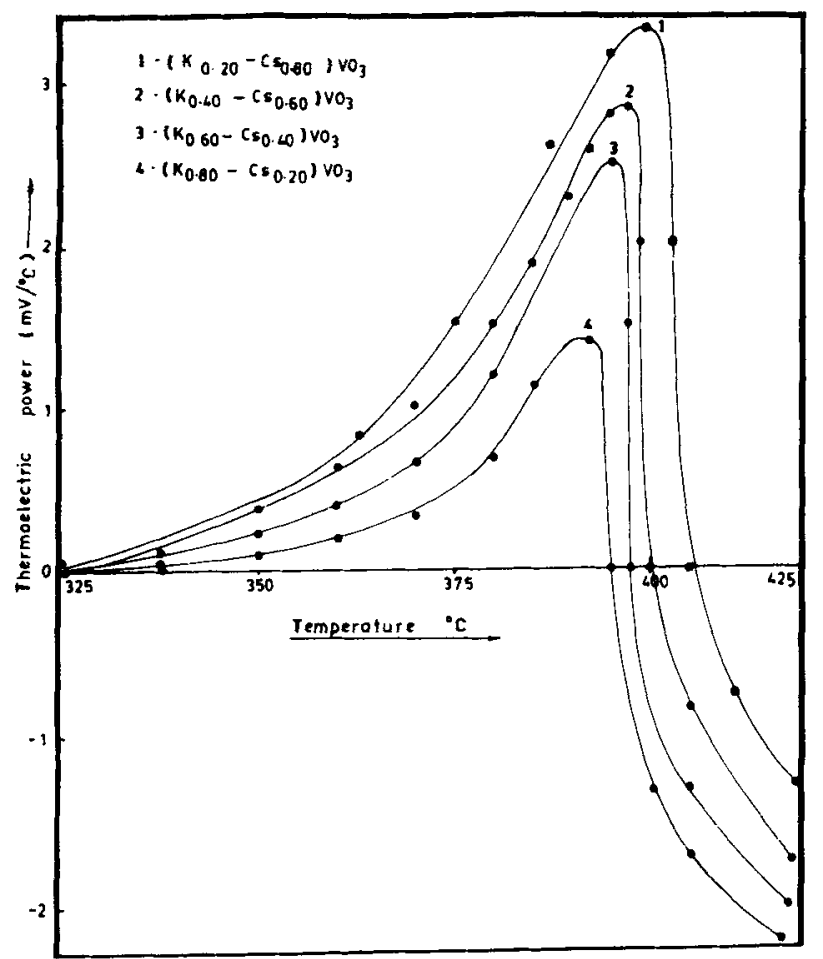

Figure 2. Variation of thermoelectric power $\left(\mathrm{mV} /{ }^{\circ} \mathrm{C}\right)$ with temperature $\left({ }^{\circ} \mathrm{C}\right)$. 


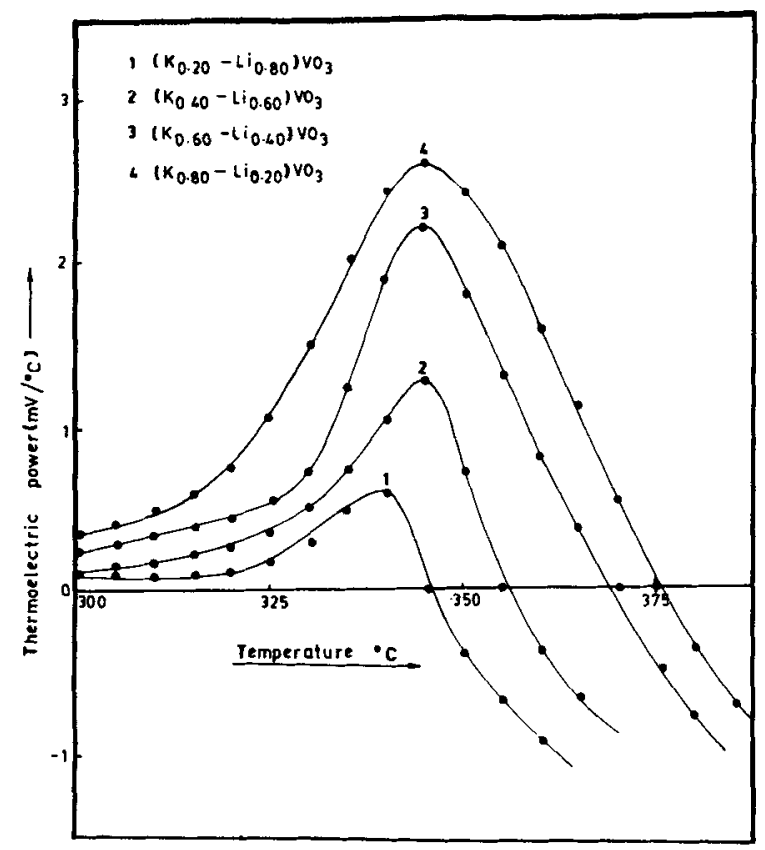

Figure 3. Variation of thermoelectric power $\left(\mathrm{mV} /{ }^{\circ} \mathrm{C}\right)$ with temperature $\left({ }^{\circ} \mathrm{C}\right)$.

direction. It was also observed that the sign of $S$ was positive in the ferroelectric region of the samples indicating that the majority charge carriers were holes. However, the sign of $S$ was reversed at a certain temperature, which indicated the phase transition temperature of the respective samples. The negative sign of $S$ in the paraelectric region of the samples revealed that the majority carriers were electrons and the samples became $n$-type semiconductors. It can therefore be concluded that the number of holes and electrons is the same at the transition temperature.

The $S$ value of $\mathrm{KVO}_{3}, \mathrm{CsVO}_{3}$ and $\mathrm{LiVO}_{3}$ showed sharp changes at phase transition temperatures of $320^{\circ} \mathrm{C}, 348^{\circ} \mathrm{C}$ and $400^{\circ} \mathrm{C}$, which were the Curie temperatures for these samples respectively and they were in good agreement with those investigated by us using hysteresis loop method (Sawyer and Tower 1930). Also the transition temperatures of the respective solid solutions were $405^{\circ} \mathrm{C}, 400^{\circ} \mathrm{C}, 395^{\circ} \mathrm{C}$ and $390^{\circ} \mathrm{C}$ for $\left(\mathrm{K}_{x}-\mathrm{Cs}_{1-x}\right) \mathrm{VO}_{3}$ and those for $\left(\mathrm{K}_{x}-\mathrm{Li}_{1-x}\right) \mathrm{VO}_{3}$ they were $345^{\circ} \mathrm{C}, 356^{\circ} \mathrm{C}, 370^{\circ} \mathrm{C}$ and $375^{\circ} \mathrm{C}$ for $x=0 \cdot 2,0 \cdot 4,0.6$ and 0.8 , respectively.

\section{Conclusions}

(i) The thermoelectric power of $\mathrm{KVO}_{3}, \mathrm{CsVO}_{3}, \mathrm{LiVO}_{3}$ and their solid solutions, $\left(\mathrm{K}_{x}-\mathrm{Cs}_{1-x}\right) \mathrm{VO}_{3}$ and $\left(\mathrm{K}_{x}-\mathrm{Li}_{1-x}\right) \mathrm{VO}_{3}$ with different concentrations of $\mathrm{KVO}_{3}$ shows a sharp change of the phase transition temperature, which indicates the ferroelectric curie temperatures of the respective samples.

(ii) The transition from a ferroelectric to a paraelectric phase is accompanied by change in sign of the thermoelectric power. 


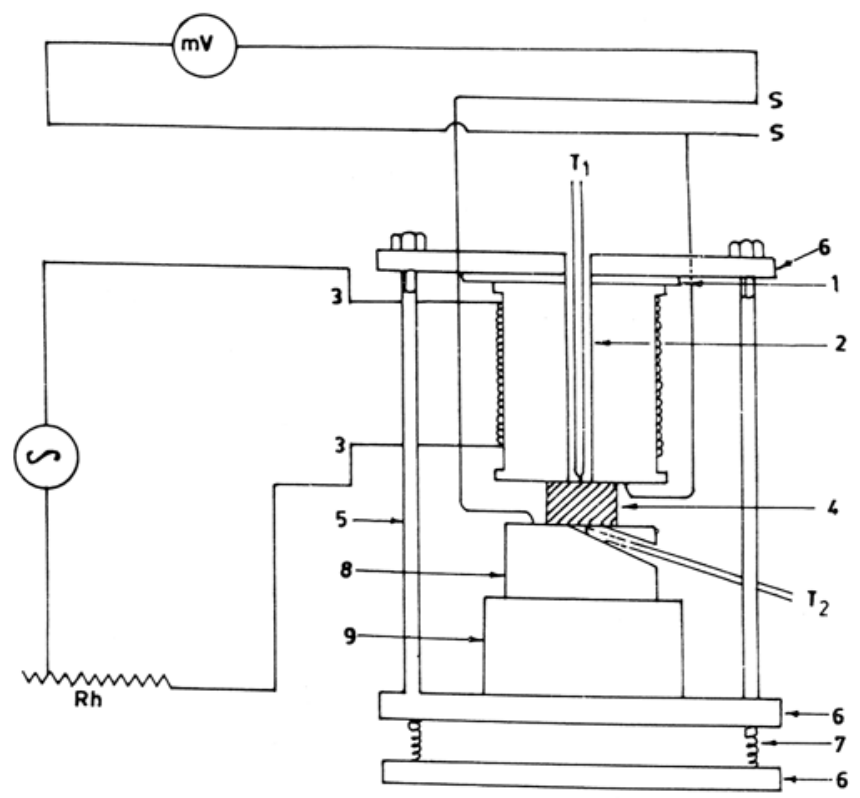

(1- Mion sheet. 2,8. Hard metal electrodes..

3. Subneater leads.. 4 - Sample pellet.. 5 - Metal rod.

6. Metal plates., 7 - Spring.. 9 - Metal block.,

$T_{1}, T_{2}$ - Thermocuples., $S$ - Silver wires welded at Ag electrodes.)

(a)

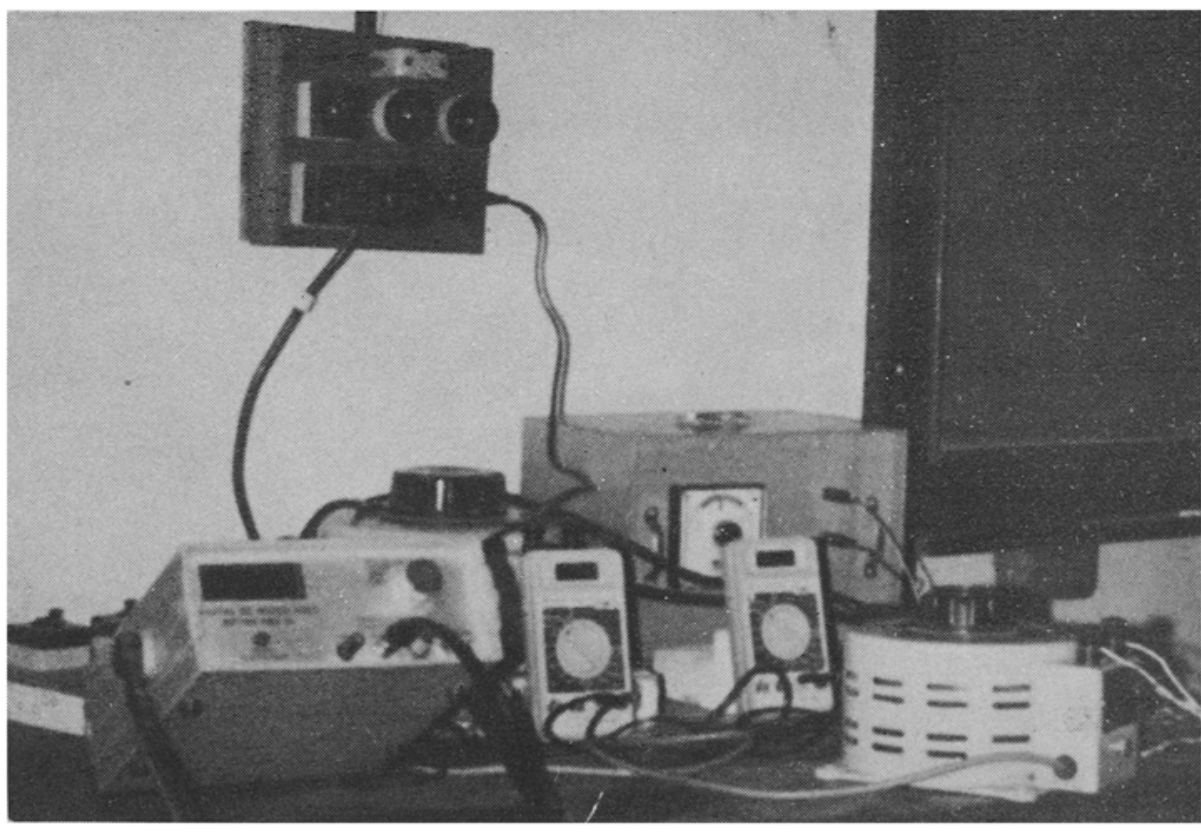

Figure 4. (a) Schematic diagram of sample holder for the measurement of thermoelectric emf and (b) experimental set-up used for the thermoelectric measurement. 
(iii) Addition of $\mathrm{KVO}_{3}$ in $\mathrm{CsVO}_{3}$ as well as in $\mathrm{LiVO}_{3}$ affects the thermoelectric properties of these materials. The increase in concentration of $\mathrm{KVO}_{3}$ decreases the thermoelectric power for the solid solutions $\left(\mathrm{K}_{x}-\mathrm{Cs}_{1-x}\right) \mathrm{VO}_{3}$. However, an increase in $\mathrm{KVO}_{3}$ concentration increases the thermoelectric power for the solid solutions $\left(\mathrm{K}_{x}-\mathrm{Li}_{1-x}\right) \mathrm{VO}_{3}$.

(iv) The concentration mode is p-type in the ferroelectric region and $n$-type in the paraelectric region.

\section{References}

Austin I G and Mott N F 1969 Adv. Phys. 1841

Bloem J 1956 Philips Res. Rep. 11273

Bosman A J and Van Daal H J 1970 Adv. Phys. 191

Gaur K and Lal H B 1985 J. Mater. Sci. 203167

Gaur K and Lal H B 1986 J. Mater. Sci. 212289

Granville J W and Hogarth C A 1951 Proc. Phys. Soc. (London) B64 488

Gupta S, Yadava Y P and Singh R A 1987 Z. Natur Forsch Tell A (Germany) A42 577

Heikes R R 1961 Thermoelectricity (New York: Wiley Interscience) p. 45

Sawyer C B and Tower C H 1930 Phys. Rev. 35269

Srivastava S K, Singh D R and Rastogi C L 1990 J. Pure Appl. Phys. 28321

Yadav Y B, Cook R L and Wanklyn B M 1985 J. Mater. Sci. Lett. 4224 